\title{
Shape Memory Effect of Keratin Fibers
}

\author{
Xueliang Xiao and Jin-lian Hu \\ Additional information is available at the end of the chapter \\ http://dx.doi.org/10.5772/intechopen.77248
}

\begin{abstract}
In the past thousands of years, keratin fibers were only considered as textile fibers for excellent fiber performances, such as high strength, acceptable elasticity, good thermal insulation, etc. Only recently, some indications have been obtained that keratin fiber may be a smart natural material that may subvert people's perception of this matter. The smart attribute displays shape memory effects (SMEs) responsive to many types of stimuli including water, heat, coupled water-heat, redox agents, UV light, etc. These smart functions of keratin fibers are found to be the result of three structural components: crystals, hydrogen bonds (HBs), and disulfide bonds (DBs) among intra- and inter-keratin macromolecules. In this chapter, keratin fibers (such as camel hair) were employed for investigating their SMEs under five types of stimuli, in which the HBs, DBs, and crystals were characterized separately, as well as the fiber shape fixation and recovery ratios, respectively. The whole test results indicated that keratin hair fiber is a type of shape memory polymer and the related SME depends on the contents of the HBs, DBs, and crystalline phase inside the hair.
\end{abstract}

Keywords: keratin fiber, shape memory effect, stimulus-responsive behavior

\section{Introduction}

Shape memory polymers (SMPs) are a kind of smart polymers from the viewpoint of their stimuli-responsive behavior adapting our human demands. SMPs have a general net-pointswitch structure [1] where net-points determine the permanent shape and reversible switch leads to the temporary shape. Most SMPs show one stimulus in the current existing cases although there are a number of recent reports of triple stimuli of SMPs [2-5]. Nowadays, people begin to realize the importance of developing multi-stimuli-responsive SMPs; for example, Schattling [6] addressed the rising importance of multi-stimuli polymers that are, 
namely, "all-in-one talents" in many areas, like comparable natural adaptability in life sciences and the parallel writing of information to give a dramatic increase of memory density in information technology. The authors believed that a polymer with one more stimulus can show a higher degree of control for more free choice and higher level of intricacy, in turn to improve the adaptability of the polymer in diverse environments with improved smart functions. Nevertheless, the development of such high intelligent polymers requires novel sciences and technologies that might go beyond our general imagination. Conversely, wisdom is encountered anywhere in nature, and many tricks can be learned even from a subject that is fairly common like a keratin animal hair [7]. For instance, a dry woolen yarn shows an interesting shape memory effect (SME) when it is immersed in water with reported $20 \%$ contraction after processes of being treated by $\mathrm{NaHSO}_{3}$ dilute solution and pre-stretched with $20-30 \%$ of strain [8]. The contraction phenomenon is believed to be a dual stimulus SME of woolen yarn responsive to water and redox.

For thousands of years, animal hair (a kind of keratin fiber) has been merely considered as a textile fiber with outstanding performances for its excellent elasticity and thermal insulation. These outstanding properties are ascribed to the keratin hierarchical structure with macroand microfibrils and helical coils, being wrapped by cortex and cuticles at the outside of the hair as shown in Figure 1a. In addition, the structural components of hydrogen (HB) and disulfide (DB) bonds between macromolecules in crystals and amorphous regions play key roles in the properties, as shown schematically in Figure 1b. HB is based on electrostatic attraction between opposite polar molecules, namely, dipole-dipole attraction, which in fact is not a true bond. Conversely, DB as a covalent cross-link for keratin molecules (can be characterized by Raman spectroscopy) [10] determines the elasticity of the hair [11, 12]. The HBs and DBs in

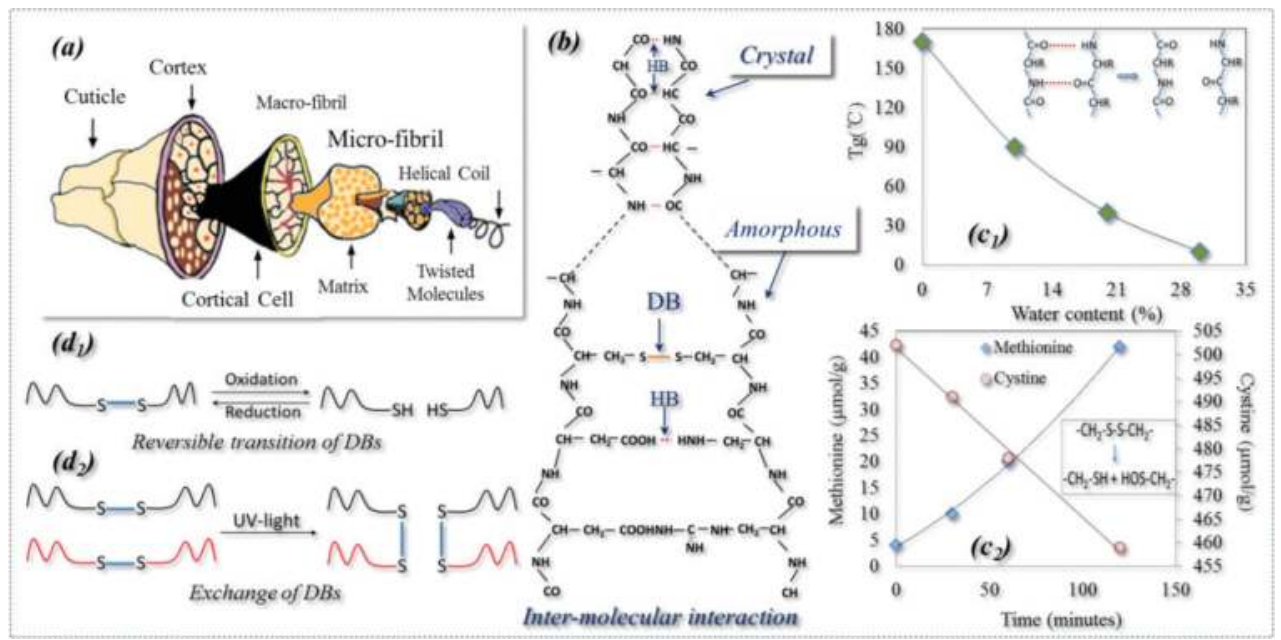

Figure 1. Components and properties of a wool fiber: (a) wool hierarchical structure from the cuticle to helical coils; (b) HBs, DBs, and crystals in wool macromolecules; $\left(c_{1}\right)$ water content influencing wool glass transition temperature; $\left(c_{2}\right)$ reducing reaction influencing amount of DBs. Schematic illustration of reversible reaction of DBs: $\left(\mathrm{d}_{1}\right)$ in redox reaction and $\left(d_{2}\right)$ under UV light radiation [9]. 
hair amorphous regions were reported reversible under certain conditions, such as processed with water, heat, and redox agents [13,14], respectively; these materials can result in conversion of keratin fiber with temporary and permanent shapes macroscopically [15]. Moreover, in Figure 1b, the schematic illustration showed the crystallinity of wool (around 40\%) difficult to be cleaved, and the crystalline phase was studied by some textile scholars using X-ray diffraction $(\mathrm{XRD})$ technique that such phase is composed of dense regular HBs [16-18].

Regarding the HBs acting as dense physical cross-linking in polymers, they perform as hard polymer crystal phase that is difficult to cleave. Such cross-linking is well known in SMPs, and the regular dense HBs are net-points in many water- and UV-induced SMPs, in which HB-based crystal acts as stress-stored net-point for shape recovery from deformation [19, 20]. When the cross-linking is not strong enough that may be reversible between decomposition and re-formation under certain reversal conditions like heat and moisture [21-23], this kind of cross-linking can be used as switch unit in SMPs. As to the keratin fiber, for example, the glass transition temperature $\left(T_{8}\right)$ of wool, was found to decrease when its water content is increased (Figure 1c $_{1}$ [24], this is because of a large amount of HBs that are collapsed in the amorphous area under the penetrated aqueous molecules. In turn, the collapsed HBs were also observed to re-form in the amorphous area of wool when the water content is decreased under drying condition. Similarly, such kind of HBs performs reversible behavior with and without water alternatively [25]. Therefore, it can be confirmed that HBs act as a key role in water-/moisturedriven shape memory behavior [7, 22, 26]. In addition, a poly(ethylene glycol) (PEG)-based polyurethane was observed with a water-induced SME, where regular dense HBs were found to be dissolved by aqueous molecules due to the collapse of HBs [27]. When the molecular weight of PEG is increased, a copolymer with PEG molecules was synthesized with a shape recovery rate of $99 \%$ using $\mathrm{HB}$ as switch [28]. A simple composite with controllable triple SME supramolecular was also developed successfully using HB as switch between macromolecule branches and mesogenic units [29] in which the HBs can improve the broad and independent control of $T_{g}$ and density of cross-linking [30].

For covalent bond of DB, it can be collapsed either when a reductant agent is used to react with such bond, and this can be reflected through the amount of cysteine and methionine with opposite tendency during the reducing reaction, as shown in Figure $\mathbf{1} \mathbf{c}_{2}[31]$. Here, the covalent bond of DB can be broken into two thiol groups after the reducing reaction. In turn, the two thiol groups can be reversible to regenerate a DB after an oxidation reaction, such as in a $\mathrm{H}_{2} \mathrm{O}_{2}$ solution [32]. DB, in this case, performs a kind of dynamic bond like HB responsive to redox agents or high-energy light triggers that can act as switch role in some SMPs. Figure $\mathbf{1 d}_{1}$ shows the reversible behavior taking place between thiol groups and DBs [33]. The reversible behavior was reported in many responsive polymer capsules, micelles, and gels for many applications like drug delivery or self-healing [34-36]. As to the self-healing, DB shows another applied type of reversibility. A specific polymer with DB can be driven for self-healing by triggers like UV radiation for exchange reaction, and an increase of DBs shows an increased healing ability, as shown in Figure $\mathbf{1 d}_{2}$ [37-40]. So far, two references were found, one thermal-responsive SMP using semicrystalline as net-point and DBs for covalently network were used to cross-link for self-healing under UV light [41]. In another reference, a SMP with DBs was reported using DB as switch for SME when responsive to redox, cellulose 
derivatives in the polymer were used with cross-linked mercapto groups [42]. Strictly speaking, the report of using DBs as net-points in SMPs has not been found yet; however, the covalent bonds are strong enough to be stable under certain conditions that manifest high opportunity to be net-points in SMPs such as in wool hair. The DBs in wool as net-points can control the wool's elasticity significantly under general environmental factors [11, 12]. In a conclusion, DBs as reversible component could be switches responsive to redox, showing selfhealing capability in some polymers, when reversible reactions take place between keratin macromolecules under specific (UV radiation) conditions [43].

So far, the three components in polymer (crystalline phase, HBs, and DBs) may form or tailor many types of combinations for a variety of SMEs and performances [44]. It is interesting to develop a kind of multi-responsive SMPs through studying and mimicking the components, structures, and related shape memory functions of keratin fiber. This would inevitably enrich the super intelligent family of SMPs.

\section{Water-sensitive shape memory behavior of keratin fiber}

As is known, a dry mature animal hair usually has three layers, i.e., a porous medulla in the hair center, a middle layer of cortex, and a surface layer of tiles-overlapping scales (around $0.5 \mu \mathrm{m}$ of thickness). In this section, as shown in the SEM images in Figure 2a, three kinds of keratin hairs evidence the three-layer structure under a dry state. The volume ratio of the cortex and the texture of the medulla result in a bit difference of the configuration of three keratin fibers.

The central medulla is a form of porous medium made of biodegradable cellular materials. The porous structure benefits the warmth retention of keratin hair due to the stored still air. The middle layer of hair cortex accounts for the main volume of the hair and provides the main strength to deformation when facing external force. A two-phase model was once proposed to interpret the hair context that employs crystalline phase and matrix (two-phase) to simplify the hair $[45,46]$. In water, the hair cortex swells evidently, as shown in Figure 2a. This means that the aqueous molecules can penetrate easily into the matrix phase of the cortex, resulting in the thicker of wet hair with the narrower of the central medulla in comparison with dry hair. Here, a large amount of reversible polar groups in amorphous regions of the hair can interact with penetrated aqueous molecules based on the two-phase model. The penetration and interaction of aqueous molecules with $\mathrm{HBs}$ can enlarge the space of neighboring polypeptide chains by breaking $\mathrm{HBs}$ between groups of $\mathrm{N}-\mathrm{H}$ and $\mathrm{C}=\mathrm{O}$ at adjacent polypeptide branches. The cortex volume is observed to increase with diametral swelling from the macroscopic point of view, which relates to the quasi-plastic distortion by means of changing the matrix phase of weak HBs between macromolecule branches.

With respect to shape memory behavior, as shown in Figure $\mathbf{2 b}$, a mature camel guard hair shows a straight smooth configuration along its axis. The innate shape was deformed manually with an entanglement of the camel hair onto a circular plastic bar and immersion of the deformed hair into the cooled water. After a certain period of deformed hair in water, it is believed that the hair was plasticized and wrapped temporarily on the bar. A drying process gives rise to the hair in helical profile under a free state. A recovery process was given to the 


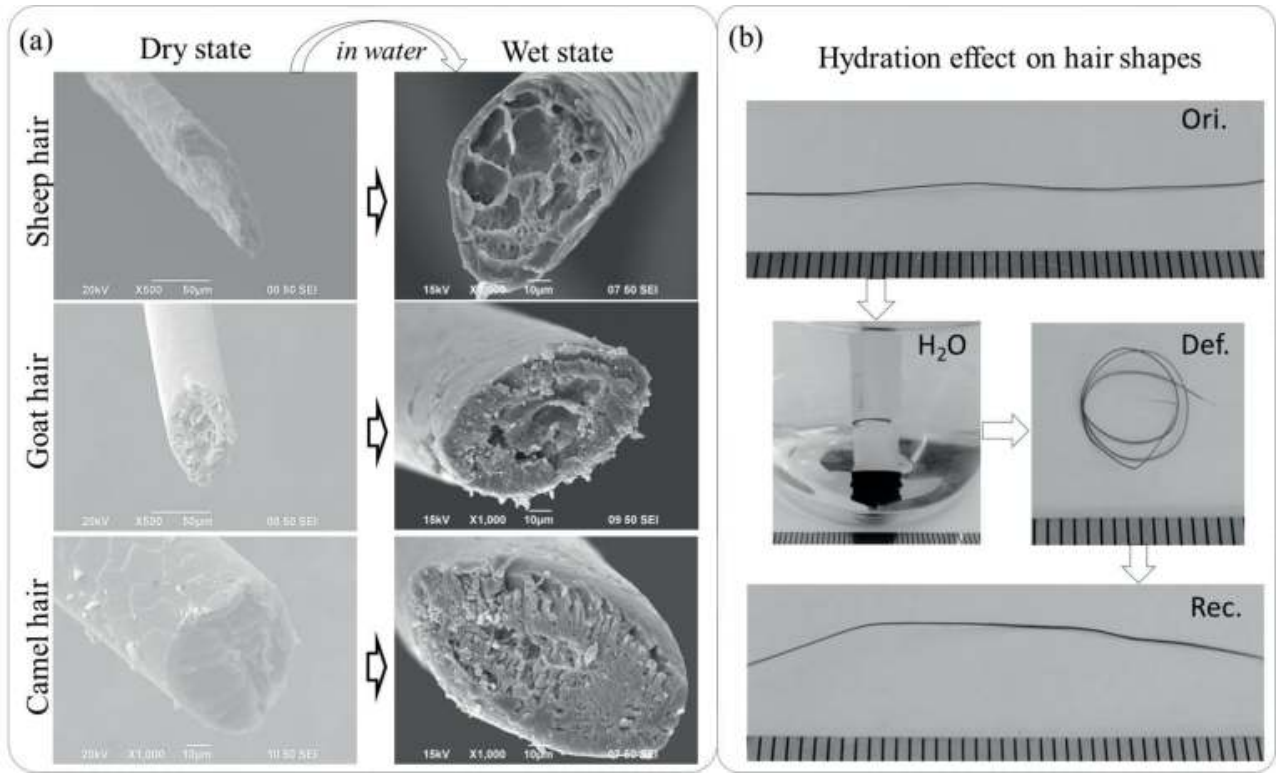

Figure 2. Hydration of three animal hairs: (a) cross sections of dry and wet sheep, goat, and camel hairs in SEM images and (b) demonstration of the water-sensitive shape memory behavior of a typical keratin fiber, camel guard hair, at the innate shape, temporarily fixed shape after hydration, and recovered shape after in water. Note: All figures were obtained at $20^{\circ} \mathrm{C}[7]$.

deformed configuration that the spatial structure recovers back to a straight shape soon after the deformed hair was put in water again. The recovery process and related dynamics of the deformed hair in water can refer to the author's work [7]. In comparison, the recovered camel hair and innate hair reveal almost the same shape without any spatial residues.

Figure 3 shows a quantification measure of water-stimulated SME of keratin fibers, in which the hair SME was investigated using the variation of folded hair in triangle into a certain angle $\left(\theta_{s}\right)$ under the transition of hair states (dry $\leftrightarrow$ wet). Here, a perfect SME of hair displays the variation of deformed angles of " $\theta=180^{\circ} \rightarrow \theta=\theta_{s}^{\circ} \rightarrow \theta=180^{\circ}$ " for the aqueous molecules to penetrate into and remove out of hairs. Nevertheless, the ideal (100\%) shape fixation and recovery cannot be realized for most natural keratin fibers, because the fibers show non-consistent network and phases between net-points. Therefore, two variables are used to justify the SME of keratin hair, i.e., $\theta=\theta_{f}$ and $\theta=\theta_{r}$ standing for the fixed shape angle and recovered shape angle, respectively. The shape fixation ratio $(R$, Eq. (1)) and shape recovery ratio $(R$, Eq. (2)) [47] are thereafter derived on the basis of the two measured angles:

$$
\begin{aligned}
& R_{f}=\frac{180-\theta_{f}}{180-\theta_{s}} \\
& R_{r}=\frac{\theta_{r}-\theta_{s}}{180-\theta_{s}}
\end{aligned}
$$


(a)
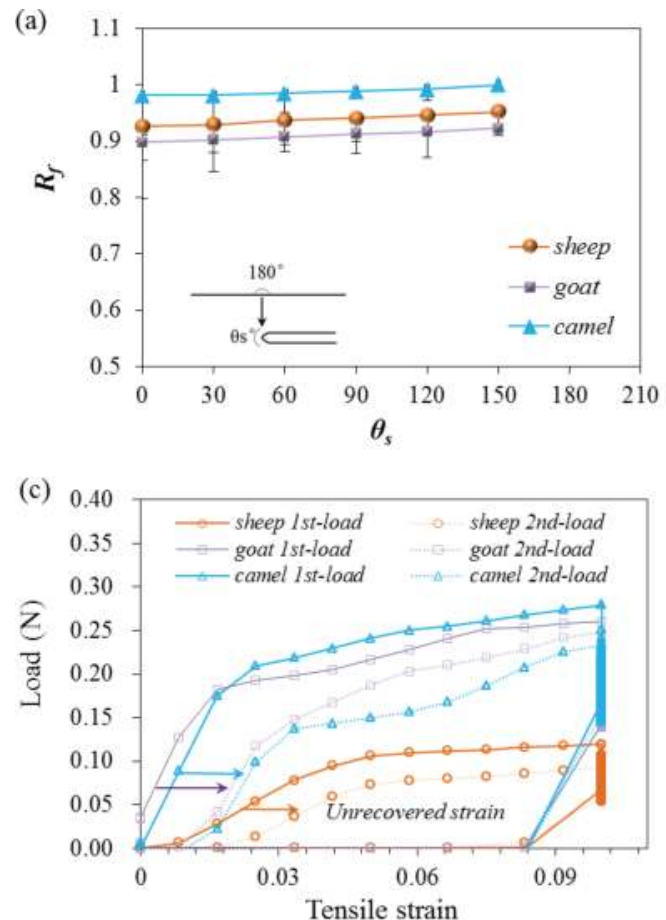

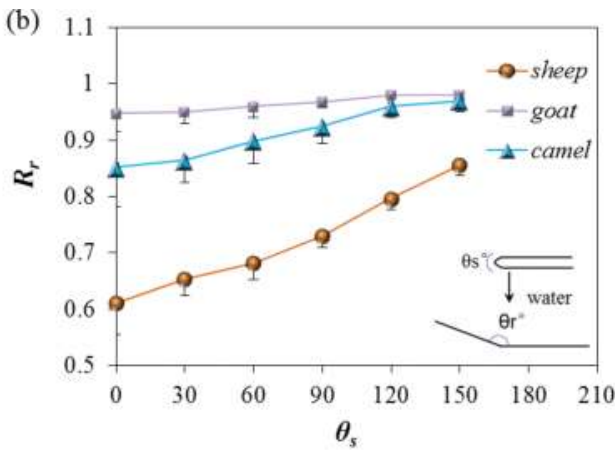

(d)

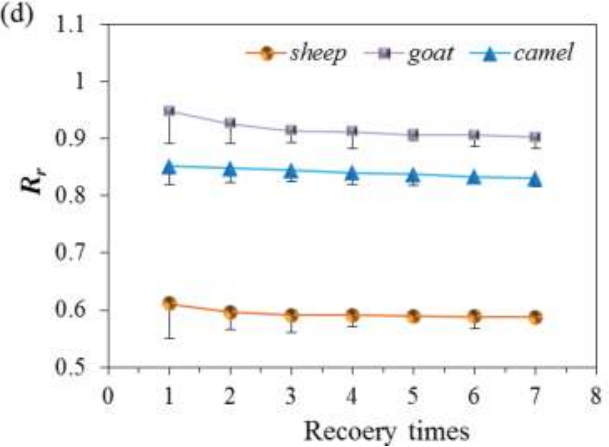

Figure 3. SME quantification of three keratin fibers responsive to water: (a) fixation ratio $\left(R_{f}\right)$ values corresponding to a set of folded angles $\left(\theta_{s}\right)$ in temporary shape; (b) shape recovery ratio $\left(R_{r}\right)$ from temporary shape to the hair innate shape (the inset illustrates the process of a camel hair from $\theta_{s}$ to $\theta_{r}$ in water); (c) two tensile cycles of three hairs responsive to water in shape recovery; and (d) $R_{r}$ values of three animal hairs from completely deformed shape to the innate shape with the cyclic number of SME program [7].

Physically, a greater $R_{f}$ value means a higher sensitive switch to be on and off, whereas a higher value of $R_{r}$ implies a better SME of hair fiber. Figure 3a shows the measured $R_{f}$ values of three animal hairs greater than 0.9 , indicating the hairs with good penetration of aqueous molecules for hydration and high ability of removal of aqueous molecules for shape fixation that both relate to HBs in amorphous regions under original and dislocated states, respectively. An increased $R_{f}$ value with an increase of set folded angles reflect that an enlarged set angle has less effect of folding process on hair molecule network, is found. In comparison with the other two hairs, the highest $R_{f}$ value of camel hair may be due to the most normalized amounts of HBs in the cortex for the best shape fixation ability. The goat hair was measured to show the highest $R_{r}$ value, indicating the hair with the best SME, as shown in Figure $3 \mathbf{b}$. An increasing tendency for the relationship of $R_{r}$ and $\theta_{s}$ is displayed for the measured keratin fibers. Among the parameters, $R_{r}$ is determined by the entropic stress from the stable net-points and mutually connected network in the hair. The measured sheep hair shows the lowest $R_{r}$ value which may be ascribed to the weakest network and least number and type of net-points for the lowest recover force. In Figure 3a, the dry sheep hair shows the thinnest cortex that may be the evidence for the reason of the lowest $R_{r}$ value. Conversely, the goat and camel hairs manifest rapid 
recovery (less than 5 seconds faster than sheep hair) that indicates the larger amount or higher density of net-points/network (either crystalline phase or DBs) for stronger recovery stress.

On the other hand, the SME of keratin fibers can also be measured through cyclic tensile loads to keratin hairs by means of measuring the recovered strain using water dropwise to the fixed stretched hair. The mechanism of this method is to identify the elastic recovery, plastic recovery, and permanent unrecovered strains, in which the set tensile strain $(0.10)$ is beyond the hair stretching yield point (around 0.05). As shown in Figure 3c, the elastic recovery takes place instantly when the elastic strain was released backward the original point and the unrecovered strain is thereafter found at the onset of the second tensile curve. In comparison to cyclic tensile curves, it is found that sheep hair also shows the most unrecovered strains after water dropwise to the hair and the goat hair still shows slightly less unrecovered strain than the camel hair, which is consistent with the triangle measurement results as shown in Figure $\mathbf{3 b}$. Especially, at the strain of 0.10 , the remarkably larger tensile loads for goat and camel hairs than the sheep hair support the assumed structure of stronger networks and more net-points of both keratin fibers. Figure 3d shows the corresponding $R_{r}$ values of three keratin hairs for seven cycles of SME programs at $\theta_{s}=0$, indicating that the shape recovery ability of keratin fiber stimulated by water is almost invariable $( \pm 5 \%)$ and the SME of keratin fiber is stable using water/moisture as stimuli.

\section{Coupled thermal-water-induced shape memory behavior}

Straightforward from Figure 4, a bundle of straight (Ori. dry) keratin fibers of camel hairs deforms into the helical structure (Def. heated) from the entanglement of raw straight hairs onto a circular steel bar (Def. dry) using a heating process. The deformed hair fibers in a free state maintained highly spiral residues (Fix. dry) that may be due to the heating on dislocated macromolecules, indicating the keratin hair with high shape fixation ability under thermal stimulus. However, the poor shape recovery ability was noted for the shape deformed hair using the same thermal stimulus [9]. In contrast, another water stimulus can recover the heatdeformed keratin hairs to their innate shape easily (Rec. wet), as shown in the recovered hair bundle in Figure 4. This means that the combination of factors of heat and water as coupled stimuli can give rise to good shape fixation ability and recovery ability; in another meaning, this can enrich the types of stimuli for keratin hair under processing and specific applications.

Here, Figure 4 shows the SEM images of camel hair in microscale, in which the variation of hair cross section under different stimuli conditions reflects the coupled stimuli of SME of the hair. For the dry raw hair, it is featured with three components (Figure 2a). A large amount of macrofibrils show parallel alignment under the hair surface scales (cortex layer), which echo the hair hierarchical structural model. The observed macrofibrils show a key role in corresponding hair SME. The heating process can remove most aqueous molecules out of fibrils such that a decreased cortex volume is noted with more porous feature in morphology. In contrast, a wetting process to the deformed hair after heating can penetrate a huge amount of water into the macrofibrils, resulting in an obvious swelling phenomenon in lateral for the cortex and a decreased volume of hair central medulla. The reason was inferred to be the plasticization 


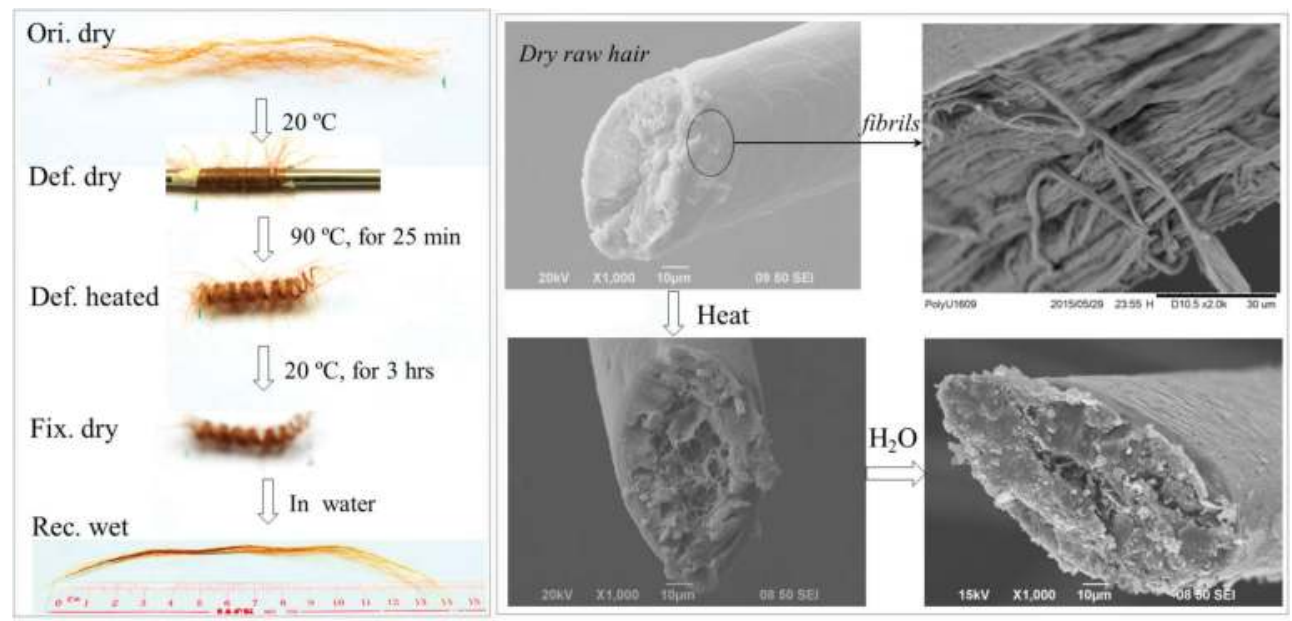

Figure 4. Demonstration of a bundle of camel hairs in coupled thermal-water-induced SME from innate shape (Ori. dry) to the heat processing on temporarily fixed shape (Def. heated and Fix. dry) and water-induced shape recovery (Rec. wet) that hydration effect of swelling is shown by SEM images of cross sections of a camel hair in dry and wet states.

of inter- and intra-fibrils, and the neighboring polypeptide chains were enlarged to their distance by means of disrupting HBs between imino and carbonyl $(\mathrm{C}=\mathrm{O}$, etc. $)$ groups at adjacent branches. The water molecules enhance the mobility of adjacent macromolecules that seems like a key to switch on the temporary shape to recover.

Compared with water-sensitive shape memory behavior for hair shape fixation (Figure 3a), heat gives rise to a relative poorer ability in this respect. However, the measured $R_{f}$ values (Eq. 1) of more than 0.75 indicate good shape fixation ability when responsive to heat, as shown in Figure 5a. The $R_{f}$ value is increased as the increase of set folded angles $\left(\theta_{s}\right)$ implies a less effect of folding process on macromolecules network when the set angle is enlarged. The highest $R_{f}$ (0.87) of hair under heating process may be ascribed to the less normalized amounts of regenerated HBs in the deformed camel hair cortex. The large error bar on each measured $R_{f}$ value indicates the uneven hair shaft used in the folding SME characterization. The measured $R_{f}$ values indicate that the deformed hairs under heating environment $\left(90^{\circ} \mathrm{C}\right.$ and $65 \%$ of $\left.\mathrm{RH}\right)$ may undergo a transfer of aqueous molecules from the hair outside into the hair cortex that relates to the regeneration of HBs at the dislocated macromolecules, as shown in the schematic illustration of Figure 5d. Moreover, Figure 5a also displays the measured $R_{r}$ values corresponding to $R_{f}$ values. The $R_{r}$ values of more than 0.87 indicate the excellent shape recovery due to the good hydration effect of deformed hair. In addition, the tendency of $R_{r}$ and $\theta_{s}$ shows an increasing relationship, in which the $R_{r}$ value is associated with the entropic stress from the stable netpoints and connected network. The lowest $R_{r}$ of the hair $\left(\theta_{s}=0\right)$ may indicate the most setting effect on hair under high humidity and temperature, meaning the least number/type of netpoints for shape recovery. Figure $5 \mathbf{b}$ shows the relationship of measured $R_{f}$ and $R_{r}$ values $\left(\theta_{s}=0\right)$ after seven cycles of shape memory tests induced by coupled thermal and water, indicating the invariable shape fixation and recovery abilities of keratin fibers (decreased $<10 \%$ ). 

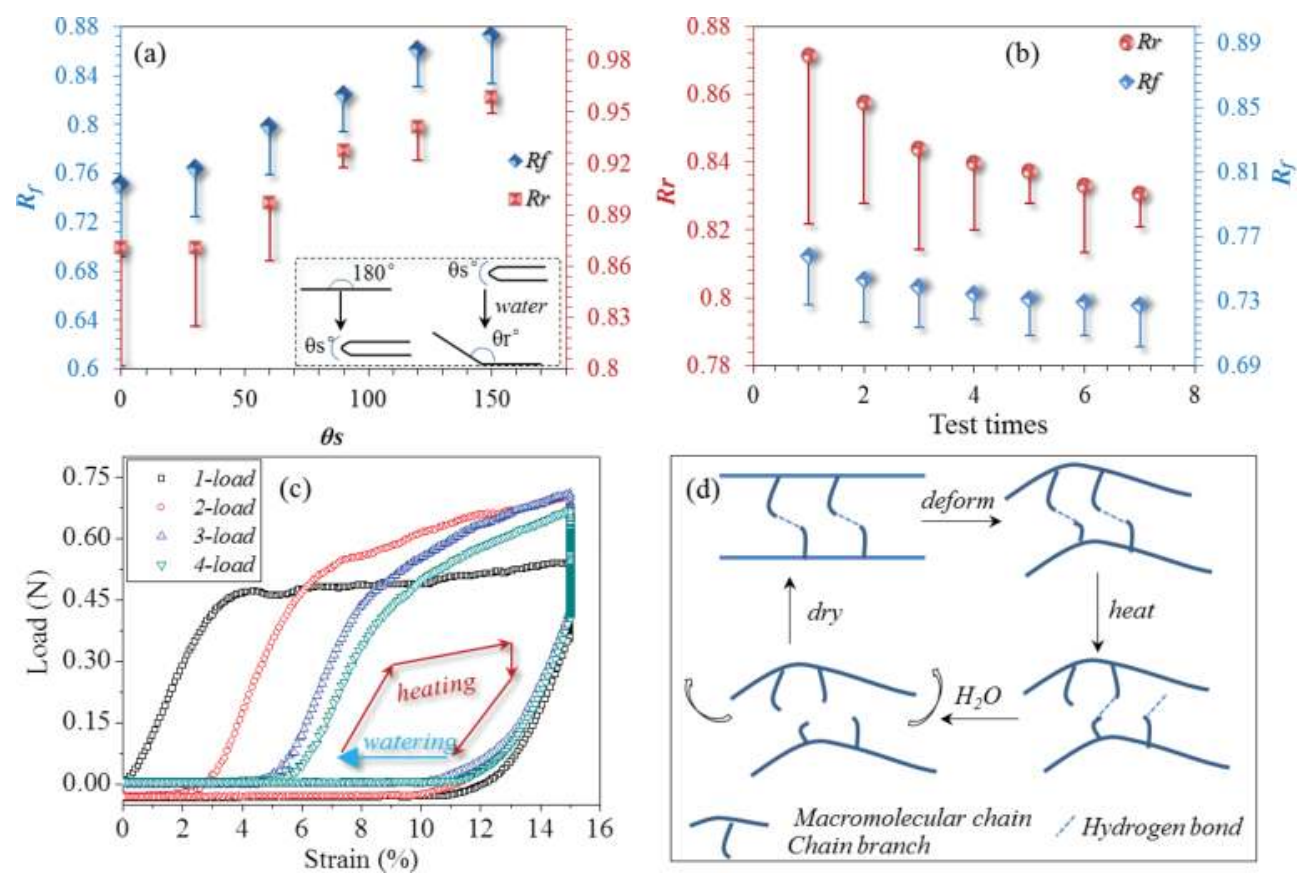

Figure 5. SME quantification of heat-induced shape fixation and water-stimulated recovery of hair fibers: (a) $R_{f}$ in temporary shape and $R_{r}$ from temporary to the innate shape corresponding to a set of $\theta_{s^{\prime}}(\mathrm{b})$ variation of $R_{f}$ and $R_{r}$ values from $\theta_{s}=0$ to the innate shape with the cyclic number of SME program, (c) cyclic tensile of an animal hair under four times of loading versus tensile strain with the coupled heat and water effects on SME of keratin fiber, and (d) schematic interpretation of coupled heat and water effects on the switch of keratin hair in macromolecular scale.

Cyclic tensile to keratin fiber is also employed here to measure the coupled stimuli SME. The 0.15 of set tensile strain is also beyond the yield point of the hair ( 0.03). The shape memory strain can be identified from the unrecovered strain and the onset of the second tensile curve. Regarding the tensile curves in Figure 5c, the largest unrecovered strain should be the "fourload" curve which means that the hair has been experiencing four cyclic tensile programs. It is found that the unrecovered strain between two adjacent loadings is getting less with an increase of loading numbers. A calculated $\sim 0.82$ of $R_{r}$ value is almost consistent with the measured results, as shown in the comparison in Figure 5(a and $\mathbf{b})$. Under the same strain of 0.15, it is found that the stretching forces of the "two-load" to "four-load" curve are remarkably greater than the "one-load" curve, supporting the viewpoint of realignment of macromolecule chains for stronger networks and net-points under conditions of tensile at $90^{\circ} \mathrm{C}$.

\section{Multi-responsive shape memory behavior}

Figure 6a gives a normally used SME program for a keratin hair. In this section, four types of stimuli were used to investigate the multi-stimuli-responsive SME behavior of camel hair. 
The related results were shown in Figure $\mathbf{6 b}$ with key images. Here, the water and redox agents as stimuli for the hair show remarkably SME, which the final recovered hair is similar with its original shape. For both factors, the aqueous molecules are believed to take an important role in SME of keratin hair [7]. In addition, as to the redox agent, the reductant ion, $\mathrm{SO}_{3}^{-2}$, has broken down some DBs between keratin macromolecules after reducing reaction; thus, one type of net-point might disappear after shape fixation. The relatively weaker shape recovery from stimulus of redox confirms the viewpoint. Here, the stimulus factors, UV light and heat, both fix camel hairs with good temporary shape with high $R_{f}$ values; however, the deformed shapes under the same environmental stimuli, i.e., poor $R_{r}$ values, which can be comparably noted from the unrecovered residues of spiral circles after recovery processes are found difficult to be recovered. Theoretically, the camel hair samples were found in much drier state caused from stimuli factors of UV light and heat, where UV light breaks down the DBs and heat leads to less structural water [37], giving rise to the temporarily deformed hairs relatively difficult to be recovered in comparison with water and redox as stimuli under the recovery duration.

Due to different growth environments and maturity, keratin hairs in fact manifest different shape memory abilities under different stimuli, depending on the hair internal contents, structure, and distribution of crystalline phase, HBs, DBs, etc. Quantitatively, according to Eqs. 1 and 2 for Figure $7 \mathbf{a}-\mathbf{d}$, the calculated $R_{f}$ values, 0.84 and 0.875 , indicate that the highest fixed strains can be achieved using water and redox as stimuli, respectively, which show higher values than the $R_{f}$ values from UV (0.65), heat (0.73), and the original state (0.65).

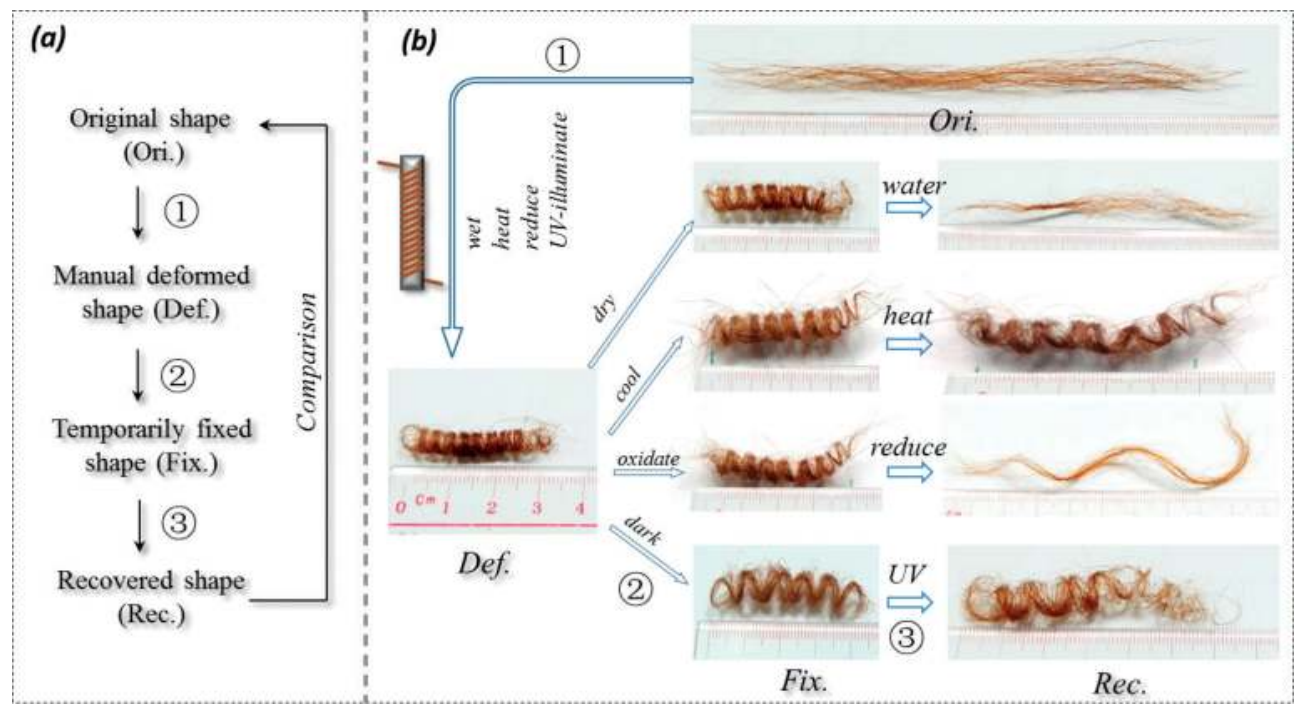

Figure 6. (a) SME investigation program and (b) observed SMEs of camel hairs under four stimuli (Ori. (original), Def. (deformed), Fix. (fixed), Rec. (recovered)) induced by water, heat $\left(85^{\circ} \mathrm{C}\right)$, redox $\left(\mathrm{NaHSO}_{3} / \mathrm{H}_{2} \mathrm{O}_{2}\right.$ solutions), and UV light $(254 \mathrm{~nm})$, respectively [9]. 

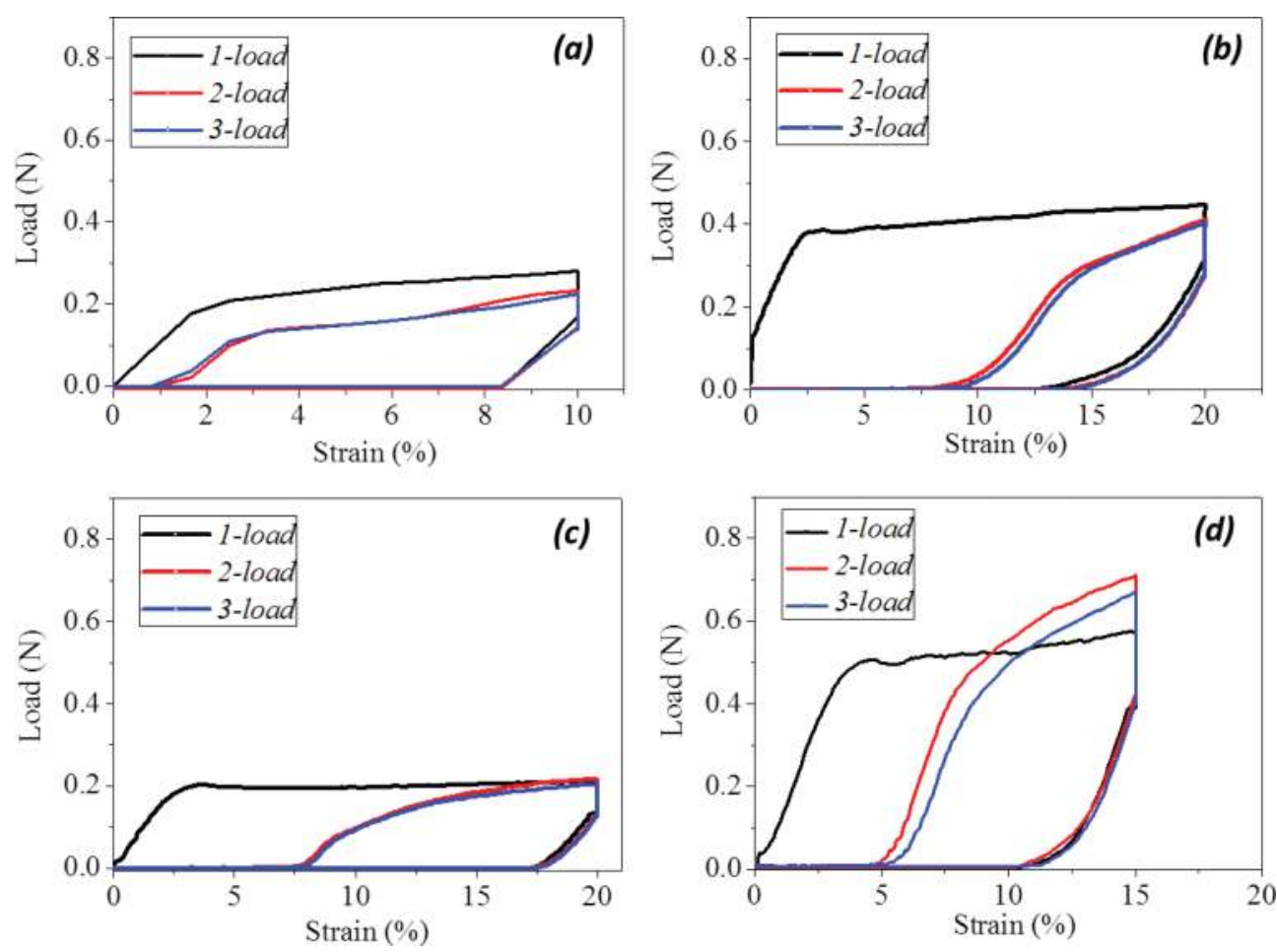

Figure 7. Experimental tests of cyclic tensile loads of camel hairs under (a) water stimulus, (b) stimulus of UV illumination (254 nm of light wave length), (c) stimulus of $\mathrm{NaHSO}_{3}-\mathrm{H}_{2} \mathrm{O}_{2}$ redox solutions, and (d) heat $\left(85^{\circ} \mathrm{C}\right)$ stimulus, respectively [9].

The reason may be the increased amount of HBs inside the hair from penetrated water molecules that act as switch for locking temporarily deformed shape. As to the $R_{r}$ values, it was noted that the best shape recovery ability is from water (0.76), followed with stimuli of heat (0.547), redox (0.525), and UV radiation (0.225), respectively. Here, all shape fixation and recovery processes were under the same tensile speed for each stimulus. Water, thus, switches on the largest amount of HBs that causes the highest mobility of macromolecules and the most recovery strains from the released stress between net-points. To some extents, stimuli of heat, redox, and UV radiation can switch on the related reversible bonds in different degrees, leaving different recovered strains as shown in Figure 7a-d. The deformed hair can also recover slightly without constrain, and stimuli are ascribed to the relaxation of polypeptide chains. However, inevitably, the unrecovered strain due to plastic tensile cannot be completed in a short time. Partial DBs can be broken down when exposure of camel hair in UV radiation promotes the shape recovery as shown in the increased $3.5 \%$ of subsequent recovered strain based on the original hair. Similarly, the stimuli of heat and redox agent both can break down related HBs that give rise to more recovered strain for higher SME, indicating that HBs should be the key switch for SME of keratin animal hairs. 


\section{Identification of net-points and switch of keratin fibers in shape memory behaviors}

Under UV radiation, reducing agent, heating, and watering conditions, the variation of internal components and structures of keratin fiber samples has been investigated from a number of characterization aspects. The DSC scanning shows two thermal absorption peaks for raw camel hairs, as shown in Figure 8a. The peak configuration displays that the first broad endothermic one appears at the temperature of $78.8^{\circ} \mathrm{C}$ with enthalpy of $270.1 \mathrm{~J} \bullet \mathrm{g}^{-1}$ that relates to the hair internal water removal from the hair [48]. The second DSC singlet appears at $230^{\circ} \mathrm{C}$, while the inset figure showing the decreased $15.1 \mathrm{~J}^{\circ} \mathrm{g}^{-1}$ of enthalpy is ascribed to the denaturation of ordered $\alpha$-helical keratin molecules [49]. This indicates that the crystalline phase is existed in animal hair, and its volume content is around $10-20 \%$ of the whole fiber, which would act as net-points in keratin hair's SME. Figure $\mathbf{8 b}$ shows five XRD patterns of camel hairs, in which the diffraction shoulders and peaks are noted for the original and arise by radiation (UV),
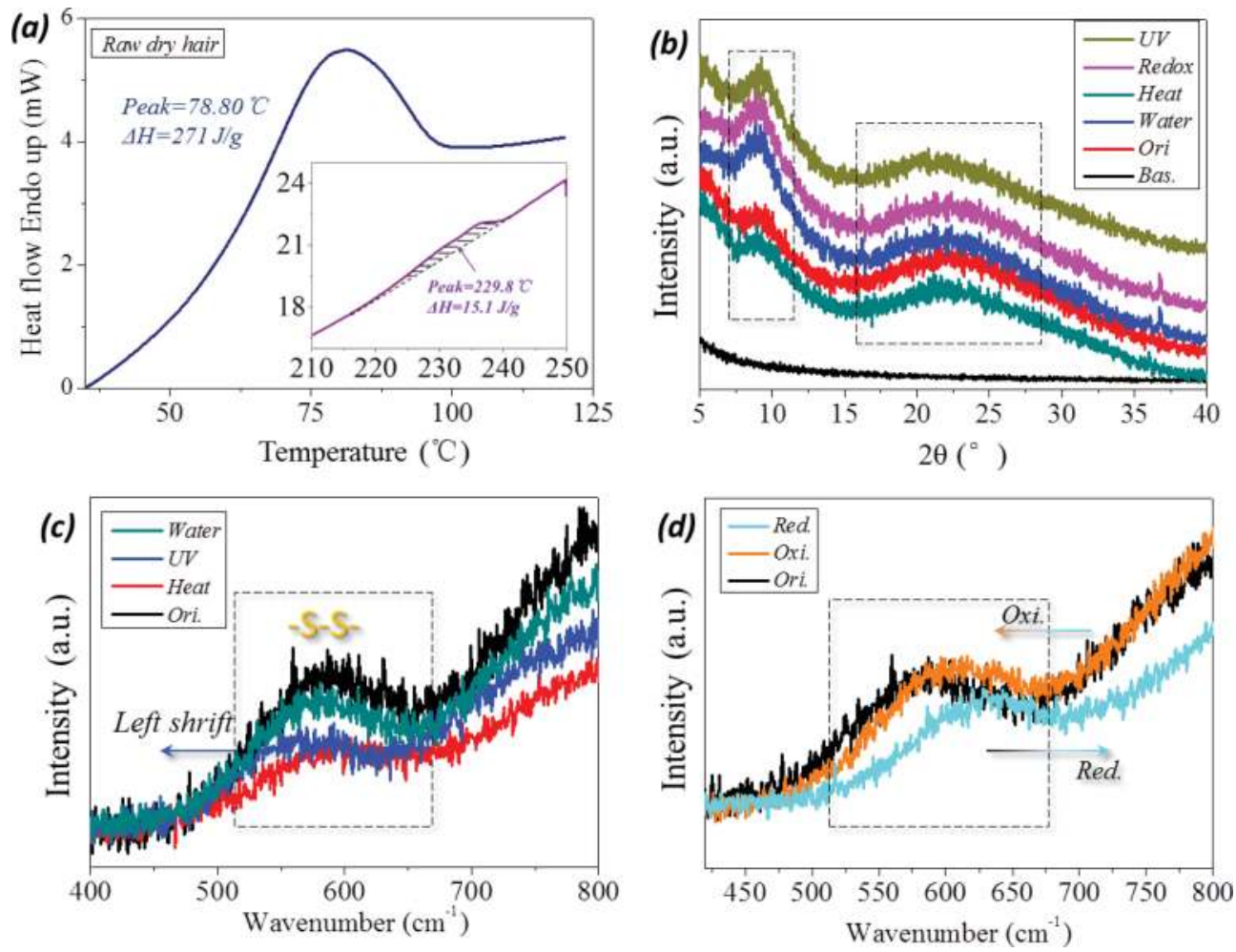

Figure 8. Identification of net-points and switches of keratin hair in SME: (a) DSC measurement of original camel hair (temperature ranging between 20 and $250^{\circ} \mathrm{C}$ ); (b) XRD characterization of camel hairs in five states: Original, wet, heated, reduced, and UV radiated; (c) Raman spectra of camel hair samples after processing by water $\left(20^{\circ} \mathrm{C}\right)$, UV radiation (254 $\mathrm{nm}$ of wave number), and heat $\left(85^{\circ} \mathrm{C}\right)$, respectively; and (d) Raman spectra of camel hairs after reducing reaction (red. $\mathrm{NaHSO}_{3^{\prime}} 1 \mathrm{M}$ solution) and oxidizing reaction (oxi. $\mathrm{H}_{2} \mathrm{O}_{2}$ dilute solution) [9]. 
reduction (redox), heating (hea.), and hydration (wet) with sharp peaks, especially the peaks at the abscissa of $2 \theta=9^{\circ}(0.98 \mathrm{~nm})$ and $21^{\circ}(0.46 \mathrm{~nm})$ that represent the $\alpha$-helix and $\beta$-keratin crystalline phases, respectively. The almost identical intensities of both characteristic peaks for camel hairs at original (Ori.), heat, wet, redox, and UV indicate the unchangeable amount of crystalline phase during the five stimuli-induced shape memory behaviors.

In detail, some processes like UV radiation and water hydration in fact have a scarce effect on the crystalline phase as shown in the invariable crystal characteristic peaks. Therefore, the existed XRD peaks for $\alpha$-helix of hair under multi-stimuli conditions indicate the net-point role of crystalline phase for related SME. In comparison to Raman spectra of camel hair samples under multi-stimuli ("wet," "UV," "heat," and redox ("red. and oxi.")) conditions, the original, wet, and heat curves can be viewed as nearly coincident in the Raman scanned regions (abscissa values of Figure 8c and d). Specifically, the DB mode is found between 500 and $580 \mathrm{~cm}^{-1}$ as a symmetrical broad characteristic peak that is associated with a few molecule conformations $[10,50]$ that are $g-g-g\left(510 \mathrm{~cm}^{-1}\right), g-g-t\left(525 \mathrm{~cm}^{-1}\right)$, and $t-g-t\left(540 \mathrm{~cm}^{-1}\right)(g$ and $t$ denote gauche and trans) conformations, respectively. The processes of heating and hydration effects on hair DBs in amorphous area show ignorable with stable chemical cross-linking that may be net-points in related SMEs, as shown in Figure 8c. Nonetheless, the abscissa of the broad peak is noted to move forward to the right and then left side corresponding to the hair samples under stimuli of reducing agent and UV radiation subsequently. This indicates that the DBs of breakage and regeneration are different for both stimuli. However, the DBs have been proven to switch on after two stimuli on keratin fibers. The Raman spectra showing keratin hair with opposite motion of peak abscissa and intensity ratio indicate the symmetrical vibration of DBs in switch on (thiol groups) and off (DBs) states, suggesting that DBs can be switched in a few stimuli-induced SMEs.

For FTIR spectra, a broad absorption band at around $3400 \mathrm{~cm}^{-1}$ is noted corresponding to the hydroxyl group and free water molecules for wet hair samples [51], as shown in Figure 9a and $\mathbf{b}$. Here, keratin hairs processed by water and reducing agent both show the deep absorption peak. Particularly, the key bands of keratin hairs such as Amide band I and Amide band II for both $\mathrm{C}=\mathrm{O}$ stretching and $\mathrm{N}-\mathrm{H}$ bending are found to shift to higher wave numbers from 1630 to $1633 \mathrm{~cm}^{-1}$ and from 1531 to $1533 \mathrm{~cm}^{-1}$. These are shown with the HBs of band in dash frame in Figure 9a and $\mathbf{b}$ and the related measured shifting values in Table 1. This shifting motion of IR curves implies that the related HBs between intermolecules are re-formed from the dislocated $\mathrm{HB}$ residues and $\mathrm{H}_{2} \mathrm{O}$ molecules during the hydration effect, being consistent with the reported results in [52]. Therefore, the penetrated $\mathrm{H}_{2} \mathrm{O}$ molecules endow the keratin fiber with two existing waters, i.e., free motional and fixed bind molecules.

Regarding the SME program in detail, four IR curves for camel hair after processing by stimuli of heating (or UV radiation) for temporary shape fixation and by wetting (or reducing agent) for the final shape recovery are labeled in Figure 9c. An evident difference of the intensity ratio of characteristic peaks of $\mathrm{N}-\mathrm{H}$ bending to $\mathrm{C}=\mathrm{O}$ stretching vibrations for the camel hair under dry (innate or heated) and wet (in water or redox agents) states, which is consistent with the comparable curves in dash frame of Figure 9a, is noted. In addition, Table 1 shows the calculated values of two characteristic peaks in wave number shifting and variation of intensity ratio along with the key SME steps under individual stimulus. In detail, the wave number of characteristic peak of $\mathrm{C}=\mathrm{O}$ stretching is shifted by increasing 

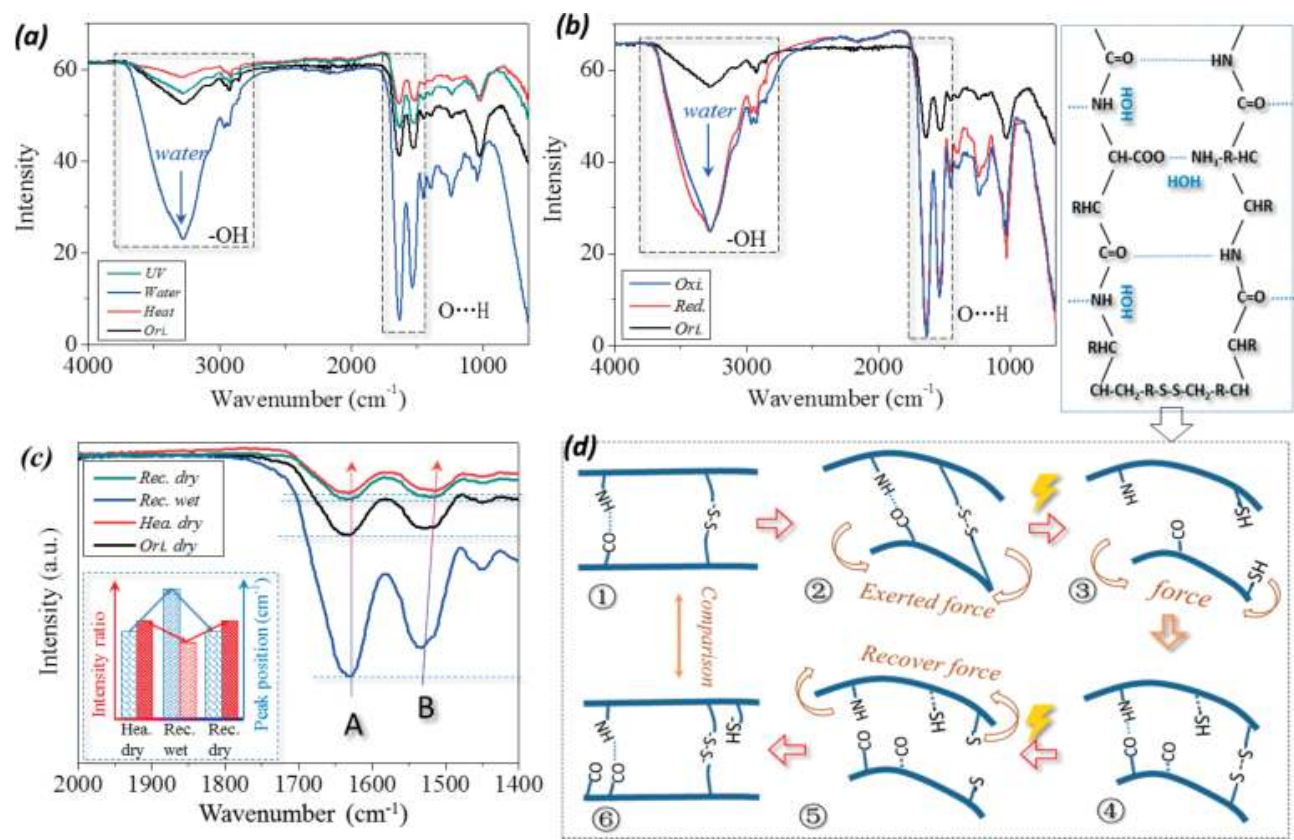

Figure 9. FTIR spectra of camel hair samples in the states of (a) Ori., water, heat $\left(85^{\circ} \mathrm{C}\right)$, and UV radiation $(254 \mathrm{~nm}$ of wave number); (b) red. and oxi. Conditions; (c) FTIR spectra for specific characteristic steps of SME program where the inset shows the IR characteristic peaks standing for HBs in open and close using shifting of peak abscissa and variation of peak intensity that also relates to the disruption and re-formation of HBs; and (d) simplification of keratin macromolecular chains and illustration of external stimuli on related switches in keratin macromolecule chains: (1) original dry state, (2) manually deformed dry state, (3) manually deformed state exposure on external stimuli, (4) temporarily fixed deformed state, (5) the fixed shape encounter with stimuli again, and (6) recovered state [9].

\begin{tabular}{llll}
\hline Hair states & \multicolumn{2}{l}{ IR peak position/wave number $\left(\mathbf{c m}^{-1}\right)$} & Peak intensity ratio \\
\hline Ori. dry/Def. wet & $1630 / 1633$ & $1531 / 1543$ & $0.979 / 0.854$ \\
Ori. dry/Def. heat & $1630 / 1634$ & $1531 / 1515$ & $0.979 / 0.994$ \\
Ori. dry/Def. UV & $1630 / 1629$ & $1531 / 1532$ & $0.979 / 0.988$ \\
Ori. dry/Def. red. & $1630 / 1633$ & $1531 / 1539$ & $0.979 / 0.834$ \\
Ori. dry/Rec. oxi. & $1630 / 1628$ & $1531 / 1532$ & $0.979 / 0.831$ \\
\hline
\end{tabular}

Table 1. Characteristic IR peaks for camel hair under different processing conditions.

2-4 $\mathrm{cm}^{-1}$ from the dry hair to wet hair; conversely, the value is reduced by $2-4 \mathrm{~cm}^{-1}$ from wet hair to dry hair. In similarity of dry and wet conversion, the wave number shifting of characteristic peak of $\mathrm{N}-\mathrm{H}$ bond move toward left and right by 8 and $16 \mathrm{~cm}^{-1}$, respectively. This reversible conversion of the keratin hair under the original, heated, wet, and dry conditions, accordingly, indicates that the HBs in macromolecules experience a reversible destruction and reconstruction process [25]. However, the wave number shifting of the characteristic 
peaks of $\mathrm{C}=\mathrm{O}$ and $\mathrm{N}-\mathrm{H}$ vibrations has not been influenced by the stimulus of $\mathrm{UV}$ radiation, indicating that $\mathrm{HB}$ is not the switch for UV-induced SME of keratin fiber.

In detail to the variation of intensity ratio for the two characteristic peaks of keratin fiber, the value for camel hair has found an increase from dry state (Ori. dry, 97.9\%) to heated (Def. hea., $99.40 \%$ ), and wet (Rec. wet, $85.45 \%$ (in water) and 83.4 and $83.1 \%$ (in redox agents)) to dry (Rec. dry, $97.42 \%)$ reverses, respectively. Figure $9 d$ interprets the conversion schematically based on the view of molecule motion corresponding to the six steps of four stimuli-induced SME program. The intensity ratios of two states, "Def. hea." and "Def. dry." (4)), almost equal one, indicate that the intensities of peaks at 1510-1535 and 1620-1640 $\mathrm{cm}^{-1}$ equal each other and the amount of carbonyl and imino groups should be the same approximately. A heating or drying process would break the balance that the excess of free water molecules would be removed out of keratin fiber [53]. For example, a tiny amount of free water molecules were attracted onto $\mathrm{N}-\mathrm{H}$ groups under normal moisture regain that may result in slightly decreased intensity ratio of characteristic peak for keratin hair at innate or deformed dry states from heated dry state (1) and (6)). The penetrated water molecules can break down HBs generated by carbonyl and imino groups (3) and (5)) when the temporarily deformed keratin hairs were soaked in water or solution. In this case, each aqueous molecule was attached onto an imino group because of polar attraction between atoms $\mathrm{N}-\underline{\mathrm{H}}$ and $\mathrm{H}-\underline{\mathrm{O}}-\mathrm{H}$, giving rise to the decreased number of discrete $\mathrm{N}-\mathrm{H}$ group for wave number of $1510-1535 \mathrm{~cm}^{-1}$ and correspondingly the evitable reduced intensity ratio for the two characteristic peaks. The temporarily locked shape from HBs was switched on by soaking process into free-constrained hair, and the shape recovered for the innate shape due to the released stress from net-points. The reversible conversion of drying and wetting of keratin hairs enable the same interaction of HBs among the hydrogen and oxygen atoms on amino, carbonyl, and hydroxyl in hairs, respectively [54]. Here, it should be noted that the interaction dynamics such as speed of responsive is not involved in the figure; the illustration only demonstrates the transformation process of each SME static step. In contrast, FTIR scanning cannot be used for characterizing the variation of DBs; thus, the SMEs induced by UV radiation and redox agents require another method to observe the variation, as referred to the DBs as switch under stimulus of redox in Figure 8d.

With respect to the tendency summary, as shown in the inset of Figure 9c, two characteristic IR peaks appearing at 1510-1535 and $1620-1640 \mathrm{~cm}^{-1}$ undergo the higher value of wave number shifting and reduced value of peak intensity ratio from the hair samples in the deformed heating/reducing or recovered wet to the final recovered dry state. The shifting and regular variation indicate that HBs in keratin fiber can lock its temporarily fixed shape by removal of internal water (heating or drying) and recover the innate shape wetting environment. This mechanism of HB implies it as the role of switch in water-, thermal-, and reducing agentsensitive SME of keratin fibers.

After SME investigation of keratin fiber (taking camel hair as a typical keratin fiber), it can be concluded that keratin fiber is an intelligent biopolymer material with different shape memory functions that can be responsive to thermal, water, redox agent, and UV radiation in varying degrees. The inherent reason depends on the structural components of keratin fiber with different amounts of crystalline phase, HBs, and DBs among keratin macromolecules. To interpret the component role in SMEs, as shown in Figure 10a (original), a twin-net-switch 


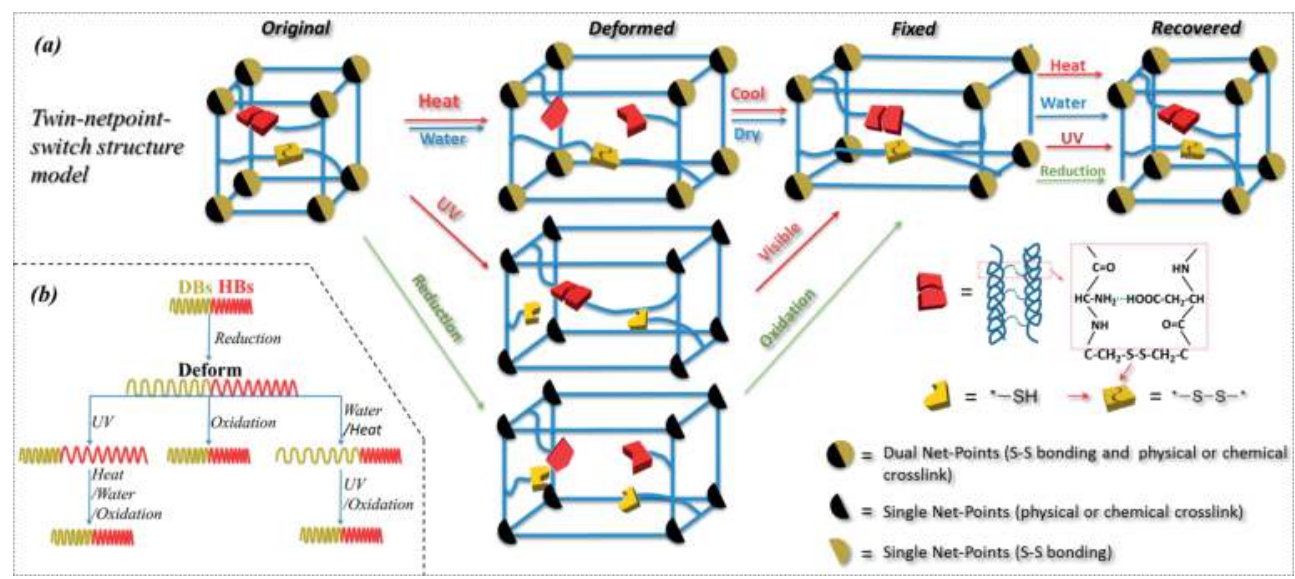

Figure 10. Multi-responsive SME mechanism: (a) a proposed structural model for different SMEs of keratin fiber; (b) the change of DBs and HBs under four types of stimuli where reducing agent can open both DBs and HBs; HBs can be regenerated using oxidant, water, and heat; and DBs can be re-formed using UV radiation and oxidant agent, respectively [9].

model is proposed that crystalline phase and DBs may be net-points and HBs and DBs may be switch, respectively. Therefore, two sets of net-points and switches for such smart biopolymer structure are, in simplicity, named as "twin-net-point-switch model." In the model, DBs act as two roles, depending on the environmental stimulus applied to the hair; for instance, DBs would be net-points for the hair in water, while they would be switch for hair in UV radiation or reducing agent. In addition, the keratin temporary shape can be programmed due to the opening of switch under a specific stimulus, and relative net-points can recover the innate shape. Figure 10a and $\mathbf{b}$ shows that HBs in amorphous region of the hair act as the single switch, whereas crystals and DBs both act as twin-net-points for stimuli of water and heat, DBs act as switch, and crystals work as single net-point for stimulus of UV radiation. Nevertheless, both bands are both switched on and off due to the water molecules and $\mathrm{SO}_{3}^{-2}$ ions for stimulus of reducing agent, indicating that they both are switch, while crystalline phase acts as single net-point without variation during SME program. With the aid of proposed model, a high possibility for developing an "all-in-one" intelligent SMP with multistimuli is believed to exist inspired from our rediscovery of keratin fibers.

\section{Conclusions}

In this chapter, keratin fiber was discussed for its SMEs responsive to various environmental stimuli, such as water, heat, coupled heat-water, UV radiation, and redox agent, respectively. The detailed mechanism was investigated using camel hair as a typical keratin fiber in molecular and structural networks. The keratin fiber with varying degrees of SME exposure on different stimuli for temporarily fixed shape recovering the innate shape was found. The calculated shape fixation ratio of more than 0.8 and recovery ratio of more than 0.5 indicate that 
the keratin fiber is a smart material stimulated by water and redox agent. The low shape fixation ratio (0.55) of keratin fiber under thermal stimulus and low recovery ratio (0.23) under UV radiation demonstrate that keratin fiber has less SMEs under such stimuli.

The cyclic tensile program of keratin fiber explains the SMEs well for each stimulus on the related switch on and off assuming net-point intact after stimuli. Some characterization approaches such as XRD and DSC of keratin fiber under five types of stimuli indicated that the crystals are invariable components during SME program. Raman spectra indicated the DBs in reversible breakage and regeneration when keratin fiber exposure on UV radiation and reducing agent, and invariation under stimuli of water and heat, indicates that DBs act as switch for stimuli of UV and redox agent and work as net-point for stimuli of water and heat. FTIR spectra of keratin fiber during SME program showed the related characteristic peaks in shifting wave number and variation of intensity ratio, indicating that HBs may act as switch for stimuli of water, heat, and redox agent. The last section proposed a structural model, namely, "twinnet-point-switch," for keratin fiber to interpret different SMEs of its exposure on different external stimuli, in which a twin-net-point/single-switch model is used for stimuli of water, heat and UV radiation, while a single-net-point/twin-switch model is used for stimuli of redox agent. This chapter for shape memory of keratin fiber is believed to have the ability to provide inspiration for manufacturing more remarkable synthetic SMPs responsive to multi-stimuli.

\section{Acknowledgements}

The authors gratefully acknowledge the financial support from the National Natural Science Foundation of China (Grant No. 51703083), the Natural Science Foundation of Jiangsu Province of China (Grant No. BK20160157), and the Hong Kong General Research Fund (RGC Project No. 15209815). In addition, the research support from Dr. Lu Jing, Mr. Wang Yifu, Miss Gui Xiaoting, and Dr. Wu You is specifically acknowledged in terms of experimental design and test, characterization and schematic drawing, etc.

\section{Conflict of interest}

Competing financial interests: The authors declare no competing financial interests.

\section{Author details}

Xueliang $\mathrm{Xiao}^{1}$ and Jin-lian $\mathrm{Hu}^{2 *}$

*Address all correspondence to: jin-lian.hu@polyu.edu.hk

1 School of Textiles and Clothing, Jiangnan University, Wuxi, P.R. China

2 Institute of Textiles and Clothing, The Hong Kong Polytechnic University, Hung Hom, Hong Kong 


\section{References}

[1] $\mathrm{Hu}$ JL, Chen SJ. A review of actively moving polymers in textile applications. Journal of Materials Chemistry. 2010;20(17):3346-3355. DOI: 10.1039/b922872a

[2] Kumpfer JR, Rowan SJ. Thermo-, photo-, and chemo-responsive shape-memory properties from photo-cross-linked metallo-supramolecular polymers. Journal of the American Chemical Society. 2011;133(32):12866-12874. DOI: 10.1021/ja205332w

[3] Wang L, Yang XF, Chen HM, Yang G, Gong T, Li WB, Zhou SB. Multi-stimuli sensitive shape memory poly(vinyl alcohol)-graft-polyurethane. Polymer Chemistry. 2013;4:4461-4468. DOI: $10.1039 /$ c3py00519d

[4] Tao Z, Peng K, Fan Y, Liu Y, Yang H. Multi-stimuli responsive supramolecular hydrogel based on $\mathrm{Fe}^{3+}$ and Diblock copolymer micelles Complexation. Polymer Chemistry. 2016;7(7):1405-1412. DOI: 10.1039/C5PY01742D

[5] Chen J, Zhang S, Sun F, Li N, Cui K. Multi-stimuli responsive supramolecular polymers and their electrospun nanofibers. Polymer Chemistry. 2016;7(17):2947-2954. DOI: 10.1039/C6PY00445H

[6] Schattling P, Jochum FD, Theato P. Multi-stimuli responsive polymers-the all-in-one talents. Polymer Chemistry. 2014;5:25-36. DOI: 10.1039/C3PY00880K

[7] Xiao XL, Hu JL. Animal hairs as water-stimulated shape memory materials: Mechanism and structural networks in molecular assemblies. Scientific Reports. 2016;7:26393. DOI: 10.1038/srep26393

[8] Hu L, Zhu Y, Huang H, Lu J. Recent advances in shape-memory polymers: Structure, mechanism, functionality, modeling and applications. Progress in Polymer Science. 2012;37(12):1720-1763. DOI: 10.1016/j.progpolymsci.2012.06.001

[9] Xiao X, Hu J, Gui X, Lu J, Luo H. Is biopolymer hair a multiresponsive smart material? Polymer Chemistry. 2017;8:283-294. DOI: 10.1039/C6PY01283C

[10] Akhtar W, Edwards H G M. Fourier-transform Raman spectroscopy of mammalian and avian keratotic biopolymers. Spectrochimica Acta Part A Molecular \& Biomolecular Spectroscopy. 1997; 53A(1): 81-90. DOI: 10.1016/S1386-1425(97)83011-9

[11] Harris M, Mizell LR, Fourt L. Elasticity of wool-Relation to chemical structure. Industrial Engineering Chemistry. 1942;34:833-838. DOI: 10.1021/ie50391a014

[12] Lindley $\mathrm{H}$. The elastic properties of chemically modified wools and their implications for theories of wool structure. Textile Research Journal. 1957;27(9):690-695. DOI: 10.1177/004051755702700902

[13] Keten S, Chou CC, van Duin AC, Buehler MJ. Tunable nanomechanics of protein disulfide bonds in redox microenvironments. Journal of the Mechanical Behavior of Biomedical Materials. 2012;5(1):32. DOI: 10.1016/j.jmbbm.2011.08.017

[14] Chapman BM. Observations on the mechanical behaviour of lincoln-wool fibres supercontracted in lithium bromide solution. Journal of the Textile Institute Proceedings \& Abstracts. 1970;61(9):448-457. DOI: 10.1080/00405007008630008 
[15] Astbury WT, Woods HJ. X-ray studies of the structure of hair, wool, and related fibres. II. The molecular structure and elastic properties of hair keratin. Philosophical Transactions of the Royal Society of London. 1934;232(788):333-394. DOI: 10.1098/rsta. 1934. 0010

[16] Samways C, Hastings GW. Radiation grafting of polysulphides to wool. Nature. 1970; 225(5233):634-635. DOI: 10.1038/225634a0

[17] Lotay SS, Speakman PT. 3-Chain merokeratin from wool may be a fragment of microfibril component macromolecule. Nature. 1977;265(5591):74-276. DOI: 10.1038/265274a0

[18] Wu B, Yi Y, Xu T, Lei H. Characterization and functions of nano-wool crystallites. International Conference on Electronic \& Mechanical Engineering and Information Technology. 2012;62(5):474-476. DOI: 10.2991/emeit.2012.311

[19] D'Hollander S, Assche GV, Mele BV. Novel synthetic strategy toward shape memory polyurethanes with a well-defined switching temperature. Polymer. 2009;50(19):44474454. DOI: 10.1016/j.polymer.2009.07.021

[20] Zhang TH, Wen ZB, Hui Y, Yang M, Yang KK, Zhou Q, Wang YZ. Facile fabrication of well-defined poly(p-dioxanone) dynamic network from metallosupramolecular interaction to access excellent shape-memory effect. Polymer Chemistry. 2015;6(22):4177-4184. DOI: $10.1039 / \mathrm{C} 5 \mathrm{PY} 00507 \mathrm{H}$

[21] Ratna D, Karger-Kocsis J. Recent advances in shape memory polymers and composites: A review. Journal of Materials Science. 2008;43(1):254-269. DOI: 10.1007/s10853-007-2176-7

[22] Luo H, Liu Y, Yu Z, Zhang S, Li B. Novel biodegradable shape memory material based on partial inclusion complex formation between alpha-cyclodextrin and poly(epsiloncaprolactone). Biomacromolecules. 2008;9(10):2573. DOI: 10.1021/bm8004726

[23] Huang WM, Yang B, An L, Li C, Chan YS. Water-driven programmable polyurethane shape memory polymer: Demonstration and mechanism. Applied Physics Letters 2005;86(11):114105-114105-3. DOI: 10.1063/1.1880448

[24] Wortmann FJ, Rigby BJ, Phillips DG. Glass transition temperature of wool as a function of regain. Textile Research Journal. 1984;54(1):6-8. DOI: 10.1177/004051758405400102

[25] Eaves JD, Loparo JJ, Fecko CJ, Roberts ST, Tokmakoff A. Hydrogen bonds in liquid water are broken only fleetingly. Proceedings of the National Academy of Sciences of the United States of America. 2005;102(37):13019. DOI: 10.1073/pnas.0505125102

[26] Fan K, Huang WM, Wang CC, Ding Z, Zhao Y. Water-responsive shape memory hybrid: Design concept and demonstration. Express Polymer Letters. 2011;5(5):409-416. DOI: 10.3144/expresspolymlett.2011.40

[27] Jung YC, So HH, Cho JW. Water-responsive shape memory polyurethane block copolymer modified with polyhedral oligomeric silsesquioxane. Journal of Macromolecular Science Part B. 2006;45(4):453-461. DOI: 10.1080/00222340600767513

[28] Liu G, Guan C, Xia H, Guo F, Ding X. Novel shape-memory polymer based on hydrogen bonding. Macromolecular Rapid Communications. 2010;27(14):1100-1104. DOI: 10.1002/ marc.200600189 
[29] Chen H, Liu Y, Gong T, Wang L, Zhao K. Use of intermolecular hydrogen bonding to synthesize triple-shape memory supermolecular composites. Rsc Advances. 2013;3(19): 7048-7056. DOI: 10.1039/C3RA00091E

[30] Ware T, Hearon K, Lonnecker A, Wooley KL, Maitland DJ. Triple-shape memory polymers based on self-complementary hydrogen bonding. Macromolecules. 2012;45(2):1062. DOI: $10.1021 / \mathrm{ma} 202098 \mathrm{~s}$

[31] Yao M. Study on the countermeasures of China-EU textile trade frictions. In: Advances in Education and Management. Berlin Heidelberg: Springer; 2011. pp. 148-153. DOI: 10.1007/ 978-3-642-23062-2_22

[32] Wedemeyer WJ, Welker E, Mahesh Narayan AM, Scheraga HA. Disulfide bonds and protein folding. Biochemistry. 2000;39(15):4207. DOI: 10.1021/bi9929220o

[33] Zheng M, Åslund F, Storz G. Activation of the OxyR transcription factor by reversible disulfide bond formation. Science. 1998;279(5357):1718-1721. DOI: 10.1126/science.279.5357.1718

[34] Yan Y, Wang Y, Heath JK. Cellular Association and Cargo Release of Redox-Responsive Polymer Capsules Mediated by Exofacial Thiols. 2011. DOI: 10.1002/adma.201101609

[35] Kakizawa YA, Harada A, Kataoka K. Environment-sensitive stabilization of core-shell structured polyion complex micelle by reversible cross-linking of the core through disulfide bond. Journal of the American Chemical Society. 2010;121(48):11247-11248. DOI: 10.1021/ja993057y

[36] Dailing EA, Nair DP, Setterberg WK, Kyburz KA, Yang C. Combined, independent small molecule release and shape memory via nanogel-coated thiourethane polymer networks. Polymer Chemistry. 2016;7(4):816. DOI: 10.1039/C5PY01464F

[37] Otsuka H, Nagano S, Kobashi Y, Maeda T, Takahara A. A dynamic covalent polymer driven by disulfide metathesis under photoirradiation. Chemical Communications. 2010;46(7):1150-1152. DOI: 10.1039/b916128g

[38] Canadell J, Goossens H, Klumperman B. Self-healing materials based on disulfide links. Macromolecules. 2011;44(8):2536-2541. DOI: 10.1021/ma2001492

[39] Fairbanks BD, Singh SP, Bowman CN, Christopher N, Anseth KS. Photodegradable, photoadaptable hydrogels via radical-mediated disulfide fragmentation reaction. Macromolecules. 2011;44(8):2444-2450. DOI: 10.1021/ma200202w

[40] Amamoto Y, Otsuka H, Takahara A, Matyjaszewski K. Self-healing of covalently crosslinked polymers by reshuffling thiuram disulfide moieties in air under visible light. Advanced Materials. 2012;24(29):3975-3980. DOI: 10.1002/adma.201201928

[41] Jakobs RTM, Ma S, Sijbesma RP. Mechanocatalytic polymerization and cross-linking in a polymeric matrix. Acs Macro Letters. 2013;2(7):613-616. DOI: 10.1021/mz400201c

[42] Dan A, Teramoto Y, Nishio Y.SH-containing cellulose acetate derivatives: Preparation and characterization as a shape memory-recovery material. Biomacromolecules. 2007;8(12): 3749-3757. DOI: 10.1021/bm7006828 
[43] Chang R, Huang Y, Shan G. Alternating poly(lactic acid)/poly(ethylene-co-butylene) supramolecular multiblock copolymers with tunable shape memory and self-healing property. Polymer Chemistry. 2015;6(32):5899-5910. DOI: 10.1039/C5PY00742A

[44] Ponyrko S, Donato RK, Matejka L. Tailored high performance shape memory epoxysilica nanocomposites. Structure design. Polymer Chemistry. 2016;7(3):560-572. DOI: 10.1039/C6PY90066F

[45] Feughelman M. A two-phase structure for keratin fibers. Textile Research Journal. 1959; 29:223-228. DOI: 10.1177/004051755902900305

[46] Feughelman M. Natural protein fibers. Journal of Applied Polymer Science. 2001;83(3): 489-507. DOI: 10.1016/B0-08-043152-6/01037-8

[47] Mendez J, Annamalai PK, Eichhorn SJ, Rusli R, Rowan SJ. Bioinspired mechanically adaptive polymer nanocomposites with water-activated shape-memory effect. Macromolecules. 2015;44(17):6827-6835. DOI: 10.1021/ma201502k

[48] Tonetti C, Varesano A, Vineis C, Mazzuchetti G. Differential scanning calorimetry for the identification of animal hair fibres. Journal of Thermal Analysis \& Calorimetry. 2015;119(2):1445-1451. DOI: 10.1007/s10973-014-4247-8

[49] Wortmann FJ, Deutz H. Characterizing keratins using high-pressure differential scanning calorimetry (HPDSC). Journal of Applied Polymer Science. 1993;48(1):137-150. DOI: 10.1002/app.1993.070480114

[50] Richard-Lacroix M, Pellerin C. Accurate new method for molecular orientation quantification using polarized Raman spectroscopy. Macromolecules. 2013;46(14):5561-5569. DOI: $10.1021 / \mathrm{ma} 400955 \mathrm{u}$

[51] Yao J, Liu Y, Yang S, Liu J. Characterization of secondary structure transformation of stretched and slenderized wool fibers with FTIR spectra. Journal of Engineered Fibers and Fabrics. 2008;3(2):1-10. WOS:000264531600003

[52] Ishida Y, Chabanne L, Antonietti M, Shalom M. Morphology control and photocatalysis enhancement by the one-pot synthesis of carbon nitride from preorganized hydrogenbonded supramolecular precursors. Langmuir The ACS Journal of Surface \& Colloids. 2014;30:447-451. DOI: 10.1021/la404101h

[53] Li G, Meng H, Hu J. Healable thermoset polymer composite embedded with stimuliresponsive fibres. Journal of the Royal Society Interface. 2012;9(77):3279-3287. DOI: 10.1098/ rsif.2012. 0409

[54] Chen S, Hu J, Zhuo H. Properties and mechanism of two-way shape memory polyurethane composites. Composite Scienec and Technology. 2010;70(10):1437-1443. DOI: 10.1016/j.compscitech.2010.01.017 
